R. D. Rashid, S. A. Jassim and H. Sellahewa, "LBP based on multi wavelet sub-bands feature extraction used for face recognition," presented at the 16th IEEE International Workshop on Machine Learning for Signal Processing, MLSP 2013, Southampton, 2013.

(C) 2013 IEEE.Personal use of this material is permitted. Permission from IEEE must be obtained for all other uses, in any current or future media, including reprinting/republishing this material for advertising or promotional purposes, creating new collective works, for resale or redistribution to servers or lists, or reuse of any copyrighted component of this work in other works. 


\title{
LBP BASED ON MULTI WAVELET SUB-BANDS FEATURE EXTRACTION USED FOR FACE RECOGNITION
}

\author{
Rasber D. Rashid, Sabah A. Jassim, Harin Sellahewa \\ The University of Buckingham, Buckingham, MK18 1EG, UK \\ rasber.rashid@gmail.com, \{sabah.jassim; harin.sellahewa\}@buckingham.ac.uk
}

\begin{abstract}
The strategy of extracting discriminant features from a face image is immensely important to accurate face recognition. This paper proposes a feature extraction algorithm based on wavelets and local binary patterns (LBPs). The proposed method decomposes a face image into multiple sub-bands of frequencies using wavelet transform. Each sub-band in the wavelet domain is divided into non-overlapping subregions. Then LBP histograms based on the traditional 8neighbour sampling points are extracted from the approximation sub-band, whilst 4-neighbour sampling points are used to extract LBPHs from detail sub-bands. Finally, all LBPHs are concatenated into a single feature histogram to effectively represent the face image. Euclidean distance is used to measure the similarity of different feature histograms and the final recognition is performed by the nearest-neighbour classifier.

The above strategy was tested on two publicly available face databases (Yale and ORL) using different scenarios and different combination of sub-bands. Results show that the proposed method outperforms the traditional LBP based features.
\end{abstract}

Index Terms - Biometrics, face recognition, discrete wavelet transform, local binary pattern, feature extraction.

\section{INTRODUCTION}

Automatic face image analysis has received much attention by the research community over the past decade and has wide-ranging application in information security, law enforcement, surveillance, and access control systems. Face recognition usually involves multi-step processes that include image normalization, feature extraction, and finally classification, which could be a one-to-one matching or a one-to-many matching scenario.

The strategy of extracting discriminant features of a face image that are robust to varying conditions is crucial to the reliability of face recognition technologies. Local binary pattern (LBP) operator was first proposed by Ojala et al. [1] as a powerful texture descriptor delivering excellent results in terms of accuracy in a number of applications such as texture analysis, motion detection, image retrieval, remote sensing, biomedical image analysis and face recognition.
Among these applications, LBP method has shown its potential in recognizing faces [2] under different illumination conditions and is one of the most popular local feature-based methods. The application of LBP in face recognition was proposed by Ahonen et al. [3].

LBPs of different resolutions can be obtained through changing the sampling radius $\mathrm{R}$ [4] or by down-sampling the original image prior to adopting the LBP operator with a fixed radius [5]. Wang et al. [6], proposed a pyramid-based multi-scale LBP approach. To begin with, multi-scale analysis is used to construct the face image pyramid using Gaussian filter into several levels then the LBP operator is applied to each level of the image pyramid to extract facial features under various scales. As a final step, all the extracted features are concatenated into an enhanced feature vector which is used as the face descriptor. A face feature extraction method using the idea of wavelet multi-resolution analysis was proposed by Liu et al. [7]. Here, low-frequency sub-bands (i.e. LL sub-bands) of several different scales are extracted as several sub-images of a subject. Then, each sub-image is divided into nine non-overlapping blocks from which LBP operator extract the characteristic spectrum and statistic histogram. In papers [6] and [7], non LL sub-bands (i.e. high-frequency sub-bands) are not used for feature extraction. This means useful information that represents some face features (e.g. significant features such as mouth, eyes) [8] may not be considered for recognition.

Face Recognition based on Haar LBP Histogram was proposed by Hengliang et al. [9]. They decomposed the face image into four-channel sub-bands in frequency domain using Haar wavelet transform and applied the LBP operator on each sub-band to extract the face features. Yi-Ding et al. [5] proposed a hand vein recognition scheme based on multi-scale LBP and wavelets. First, a hand vein image decomposed into two levels to obtain 8 coefficient matrices: $A_{1}, H_{1}, V_{1}, D_{1}, A_{2}, H_{2}, V_{2}$ and $D_{2}$. They excluded the two diagonal high-frequency components, $\mathrm{D}_{1}$ and $\mathrm{D}_{2}$, from the feature extraction process. Meanwhile, $\mathrm{A}_{1}, \mathrm{H}_{1}, \mathrm{~V}_{1}, \mathrm{~A}_{2}, \mathrm{H}_{2}$, $\mathrm{V}_{2}$ and the original image are chosen as multi-scale components from which LBP features are extracted. Finally, LBP features of all components are combined to obtain a single feature representation.

In the two papers [5] and [9], the LBP operator was applied on LL sub-bands and non LL sub-bands with no consideration for the differences in the frequency/feature ranges in these sub-bands for image features and texture. 
For example, applying a 2D wavelet transform (WT) on an image produce high-frequency sub-bands that include horizontal, vertical and diagonal features of the decomposed image respectively. Therefore, using the traditional LBP with radius 1 and 8 neighbour samples on non-LL sub-bands may capture useful as well as redundant information, while increasing the size of the overall face feature vector when compared to using LBP on the original image. This could be a problem for real-time identification and limit their usefulness for applications that require small feature representations. For example, the use of steganographic techniques, by law enforcement agencies, to communicate biometric data hidden in innocuous cover images ${ }^{1}$, increased size feature has an adverse impact on security and stegoimage quality.

This paper is, therefore, focused on developing and testing the performance of modified and reduced size LBP features in the wavelet domain by exploiting the knowledge of texture/features in the different wavelet sub-bands.

The rest of the paper is organised as follows. Section 2 presents background information on face description using multi-scale LBP. Section 3 presents the proposed face feature extraction method, while section 4 presents the experimental data and the results of our evaluation. Finally our conclusions are presented in section 5 .

\section{LOCAL BINARY PATTERNS BASED FACE DESCRIPTION}

\subsection{Local Binary Patterns}

Local binary pattern (LBP) was first proposed by Ojala et al. [1]. LBP normally refers to replacing image pixels with an 8-bit binary code that is derived from the pixel's neighbourhood. For a $3 \times 3$ block, the value of the centre pixel is subtracted from that of each of its 8 neighbouring pixels, and depending on the sign of the subtraction result 1 or 0 assigned to a bit. The generated bits for all the neighbouring pixels are then concatenated and encoded into binary strings. The derived binary strings are called Local Binary Patterns or LBP codes. The decimal value of the LBP code for the centre pixel $\left(\mathrm{x}_{\mathrm{c}}, \mathrm{y}_{\mathrm{c}}\right)$ is calculated as follows:

$$
\operatorname{LBP}\left(x_{c}, y_{c}\right)=\sum_{n=0}^{n=7} s\left(i_{n}-i_{c}\right) 2^{n}
$$

Where $n$ runs over the 8 neighbours of the central pixel, $i_{c}$ and $i_{n}$ are gray level values of the central pixel and the surrounding pixels, and the function $\mathrm{s}(\mathrm{x})$ is defined as:

$$
s(x)=\left\{\begin{array}{l}
1 \text { if } x \geq 0 \\
0 \text { if } x<0
\end{array}\right.
$$

\footnotetext{
${ }^{1}$ This is the aim of the first author's PhD research project.
}

The LBP operator $\mathrm{LBP}_{\mathrm{P}, \mathrm{R}}$ produces $2^{\mathrm{p}}$ different output values, corresponding to $2^{\mathrm{p}}$ different binary patterns formed by the $\mathrm{P}$ pixels in the neighbourhood of radius $\mathrm{R}$. It has been demonstrated that certain patterns contain more information than others. To describe the texture of the images, it is possible to use only a subset of the $2^{\mathrm{p}}$ binary patterns. Ojala et al. named these patterns as uniform patterns, denoted $\mathrm{LBP}_{\mathrm{P}, \mathrm{R}}^{\mathrm{u} 2}$. A local binary pattern is called uniform if it contains maximum two bitwise transitions from 0 to 1 or vice versa when the corresponding bit string is considered circular. For instance, 00000000 (0 transitions) and 01111110 (2 transitions) are uniform whereas 11101101 (4 transitions) and 01011011 (6 transitions) are not.

It is easy to show that within the $(8,1)$ neighbourhood there are only 58 uniform LBP-patterns, and the traditional uniform LBP histogram consists of 59 bins accounting for the 58 uniform patterns and one bin that corresponds to the set of non-uniform patterns, [3].

\subsection{Face description with LBP}

A face image can be thought of as a composition of micropatterns which can be described by LBP codes. The histogram of LBP computed over the whole face image encodes only the occurrences of the micro-patterns without giving any hint regarding their locations. In order to consider the aspect of 'shape information' of faces, Ahonen et al. [3] [10] proposed the division of face images into $m$ local regions to extract LBP histograms (LBPHs), and concatenating them into a single feature histogram. Figure 1. illustrates the concept. The histogram encodes both local texture and global shape of the face images [11]. The majority of existing works adopt the above scheme to extract LBP features for facial representation.

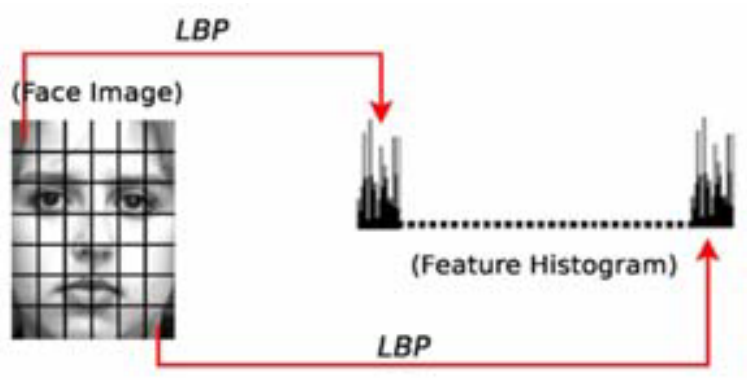

Figure1. LBP-based face description [3, 10].

\subsection{Multi-scale LBP and Wavelet decomposition}

Discrete Wavelet Transform (DWT) decomposes an image into its low- and high-frequency components at different scales. Every piece of face information (face block regions or sub-images in wavelet domain) provides different discriminative contribution and also has various 
discriminative capabilities. For example, high-frequency components have shown to be invariant to illumination changes whilst the approximation sub band is robust against facial expressions [8].

The multi-resolution representation of LBP can be implemented in two ways; either by increasing the size of the neighbourhood of LBP operator, or by down-sampling the original image prior to adopting the LBP operator with a fixed radius. It is worth mentioning that the ability of wavelets to provide a multi-scale analysis stems from the fact that it can decompose an image to a multi-resolution pyramid [5]. Combining the advantages of Haar wavelet transform and the LBP operator, LBP can be applied on the wavelet sub-bands to represent face features. Applying LBP on each sub-band can extract texture features of each subband. Combining extracted features from more than one sub-band can create final face descriptor feature vector. However, the feature vector size may be long depending on the number of blocks each sub-band is divided into.

\section{PROPOSED FACE FEATURE EXTRACTION}

In this paper, we focus on reducing the number of features that represent a face image while maintaining the recognition accuracy at the same level, or even increase, when compared to using $\mathrm{LBP}_{8,1}$ or $\mathrm{LBP}_{8,1}^{\mathrm{u} 2}$. This decision is closely related to our interest in the use of steganography for hiding biometric data in images. The number of features that represent any face image would be hugely important in determining the performance such a scheme in that it helps reduce embedding capacity requirements.

The DWT using Haar filter on face images provides adequate opportunities for reducing the size of LBP features by exploiting the structural properties of the various frequency sub-bands. For example, the LH sub-band extracts vertical features of the face image and HL sub-band extracts horizontal features of the face image.

Here we propose a new LBP representation that uses some or all the 8 neighbours of the wavelet decomposed pixels depending on the wavelet sub-bands to which the pixel belongs.

First, a given image is decomposed into four sub-bands using Haar wavelet transform. Each sub-band is divided into non-overlapping blocks of size $\mathrm{n} \times \mathrm{n}$. Then we extract LBPHs from each of the blocks of a given sub-band in the following way. We separate the 8 neighbours of a pixel into two parts, the four main neighbours $\left(\mathrm{N}_{4}\right)$ and the four diagonal neighbours $\left(\mathrm{N}_{\mathrm{D}}\right)$. Then we calculate two separate LBP codes, $\mathrm{LBP}_{4}$ and $\mathrm{LBP}_{\mathrm{D}}$ (i.e., one based on $\mathrm{N}_{4}$ neighbours and the other based on $\mathrm{N}_{\mathrm{D}}$ neighbours) at radius of 1. Then the histogram of each LBP set is calculated and the two histograms, $\mathrm{LBP}_{4} \mathrm{H}$ and $\mathrm{LBP}_{\mathrm{D}} \mathrm{H}$, are concatenated to represent the sub-block of the sub-band of the given face. Figure 2 represents locations of the four main and diagonal neighbours of the selected center pixel.
Using the proposed approach, we are able to represent LBP histograms using only 32 bins (16 bins for each of the two 4 neighbours) as opposed to 256 bins with traditional $\mathrm{LBP}_{8,1}$ or 59 bins in $\mathrm{LBP}_{8,1}^{\mathrm{u} 2}$. Also, using this approach we can extract features of a face that are very useful for recognition purpose, especially when used with non-LL subbands in the wavelet domain.
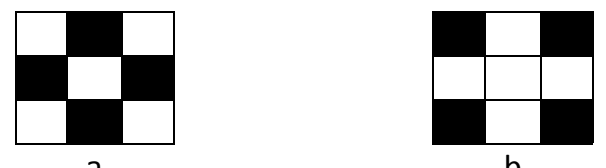

Figure 2. a) 4 main neighbour $\left(\mathrm{N}_{4}\right)$ positions, b) 4 diagonal neighbour $\left(\mathrm{N}_{\mathrm{D}}\right)$ positions

\section{EXPERIMENTAL DATA AND RESULTS}

\subsection{Databases}

We used two publicly available face databases to evaluate the accuracy of the proposed approach. The ORL database [12] contains 40 distinct subjects, each with 10 different images captured in up-right frontal position with tolerance for some tilting and rotation of up to 20 degrees with $112 \times 92$ pixel resolution. The Yale face database [13] consists of 15 individuals, where for each individual, there are 11 face images containing variations in illumination (center-light, left-light and right-light), facial expression (normal, happy, sad, sleepy, surprised and wink), and face details (with glasses and without glasses) all images with $96 \times 80$ pixel resolution.

\subsection{Evaluation Protocol}

Experiments were conducted for three different scenarios:

1. Single training image: one image per subject was selected for training and the rest used as testing. Experiments were repeated (n) times, where (n) is the number of subjects in each database and the results were averaged.

2. 50\% Training and $\mathbf{5 0 \%}$ testing: half of the images of a subject were used as training and the rest used as testing. For the ORL database, 5 images per subject were used as "training" and the remaining 5 were used as "testing"; while for Yale database, 5 images per subject were used as "training" and the remaining 6 images were used as 'testing". Experiments were repeated 10 times using randomly selected training samples. Again, the results were averaged.

3. Leave-One-Out strategy: one image at a time was taken off the database for testing and whilst the remaining images were used as training.

Nearest neighbour classification with Euclidean distance was used to calculate recognition rates. Experiments were conducted using two different numbers of blocks, $3 \times 3$ and 
$5 \times 5$ (i.e., each sub-band was divided into 9 or 25 blocks and LBPHs are extracted from each block). In all cases, we compare our results with the use of the traditional 8neighbour uniform LBP feature extractor.

\subsection{Experiments and Results}

We evaluated the proposed LBP in the wavelet domain in two scenarios. In the first scenario, $\mathrm{LBP}_{4} \mathrm{H}$ and $\mathrm{LBP}_{\mathrm{D}} \mathrm{H}$ are obtained from a sub-band at decomposition level one or two, and concatenated into a single feature histogram (i.e. $\mathrm{N}_{4} \&$ $\mathrm{N}_{\mathrm{D}}$ ). We tested the performance of the extracted features in terms of recognition accuracy. In the second scenario, we combined the concatenated feature histograms obtained two or more wavelet sub-bands. In both scenarios, LBPHs were obtained from sub-bands that were divided into either $3 \times 3$ or $5 \times 5$ blocks. Recognition rates are reported for Yale and ORL databases.

\subsection{1 $\mathrm{LBP}_{8}$ vs. combined $\mathrm{LBP}_{4}$ and $\mathrm{LPB}_{D}$ :}

Tables 1 to 4 show recognition rates of different databases and for different number of blocks that each sub-band was divided into. Results of the proposed $\mathrm{LPB}_{4}$ and $\mathrm{LBP}_{\mathrm{D}}$ based features are compared with those achieved with features based on traditional $\mathrm{N}_{8}$ uniform LBP (i.e., $\mathrm{LBP}_{8}^{\mathrm{u} 2}$ ).

It can be noticed from the results in tables 1-4, that the combined $\mathrm{LPB}_{4}$ and $\mathrm{LBP}_{\mathrm{D}}$ features of non-LL sub-bands achieve a significantly higher recognition rate than that achieved by $\mathrm{LBP}_{8}^{\mathrm{u} 2}$. However, the results show that $\mathrm{LBP}_{8}^{\mathrm{u} 2}$ based features are better suited for the approximation sub-bands than the propose features. The results of non-LL sub-bands justify the use of four-neighbours in the highfrequency wavelet sub-bands.

Table 1: Recognition rates $(\%)$ for Yale database; each wavelet sub-band is divided into $3 \times 3$ blocks

\begin{tabular}{ccccccc}
\hline $\begin{array}{c}\text { Sub - } \\
\text { band }\end{array}$ & \multicolumn{2}{c}{$\begin{array}{c}\text { Single training } \\
\text { image }\end{array}$} & \multicolumn{2}{c}{$\begin{array}{c}\mathbf{5} \text { training } \\
\text { images }\end{array}$} & \multicolumn{2}{c}{ Leave-one-out } \\
& $\mathbf{N}_{\mathbf{8}}$ & $\mathbf{N}_{\mathbf{4}}$ \& $\mathbf{N}_{\mathbf{D}}$ & $\mathbf{N}_{\mathbf{8}}$ & $\mathbf{N}_{\mathbf{4}}$ \& $\mathbf{N}_{\mathbf{D}}$ & $\mathbf{N}_{\mathbf{8}}$ & $\mathbf{N}_{\mathbf{4}}$ \& $\mathbf{N}_{\mathbf{D}}$ \\
\hline $\mathrm{LL}_{1}$ & 76.4 & 72.7 & 90.3 & 85.3 & 89.7 & 87.3 \\
$\mathrm{HL}_{1}$ & 49.3 & 63.7 & 71.1 & 84.9 & 79.4 & 91.5 \\
$\mathrm{LH}_{1}$ & 53.2 & 70.3 & 75.4 & 87.3 & 79.4 & 89.7 \\
$\mathrm{HH}_{1}$ & 18.7 & 25.3 & 28.0 & 41.4 & 30.3 & 41.2 \\
\hline $\mathrm{LL}_{2}$ & 70.4 & 67.1 & 86.2 & 84.8 & 87.9 & 86.1 \\
$\mathrm{HL}_{2}$ & 37.2 & 53.5 & 58.9 & 79.9 & 69.7 & 85.5 \\
$\mathrm{LH}_{2}$ & 43.9 & 60.9 & 66.4 & 81.3 & 75.2 & 85.5 \\
$\mathrm{HH}_{2}$ & 14.5 & 19.4 & 26.7 & 30.7 & 28.5 & 34.5 \\
\hline
\end{tabular}

Table 2: Recognition rates (\%) for Yale database; each wavelet sub-band is divided into $5 \times 5$ blocks

\begin{tabular}{ccccccc}
\hline $\begin{array}{c}\text { Sub - } \\
\text { band }\end{array}$ & \multicolumn{2}{c}{$\begin{array}{c}\text { Single training } \\
\text { image }\end{array}$} & \multicolumn{2}{c}{$\begin{array}{c}\mathbf{5} \text { training } \\
\text { images }\end{array}$} & \multicolumn{2}{c}{ Leave-one-out } \\
& $\mathbf{N}_{\mathbf{8}}$ & $\mathbf{N}_{\mathbf{4}} \boldsymbol{\&} \mathbf{N}_{\mathbf{D}}$ & $\mathbf{N}_{\mathbf{8}}$ & $\mathbf{N}_{\mathbf{4}} \boldsymbol{\&} \mathbf{N}_{\mathbf{D}}$ & $\mathbf{N}_{\mathbf{8}}$ & $\mathbf{N}_{\mathbf{4}} \boldsymbol{\&} \mathbf{N}_{\mathbf{D}}$ \\
\hline $\mathrm{LL}_{1}$ & 82.8 & 79.2 & 94.3 & 90.9 & 94.5 & 90.3
\end{tabular}

\begin{tabular}{lllllll}
$\mathrm{HL}_{1}$ & 59.1 & 72.8 & 80.6 & 94.1 & 88.5 & 96.4 \\
$\mathrm{LH}_{1}$ & 59.5 & 78.2 & 81.7 & 94.7 & 87.3 & 97.0 \\
$\mathrm{HH}_{1}$ & 22.0 & 34.8 & 29.9 & 54.4 & 29.7 & 66.1 \\
\hline $\mathrm{LL}_{2}$ & 78.2 & 77.9 & 93.6 & 91.8 & 97.0 & 93.9 \\
$\mathrm{HL}_{2}$ & 46.3 & 62.5 & 71.3 & 86.3 & 77.0 & 91.5 \\
$\mathrm{LH}_{2}$ & 52.6 & 71.5 & 77.8 & 92.6 & 84.2 & 95.2 \\
$\mathrm{HH}_{2}$ & 18.0 & 31.6 & 28.2 & 49.4 & 32.7 & 59.4 \\
\hline
\end{tabular}

Table 3: Recognition rates (\%) for ORL database; each wavelet sub-band is divided into $3 \times 3$ blocks

\begin{tabular}{|c|c|c|c|c|c|c|}
\hline \multirow{2}{*}{$\begin{array}{l}\text { Sub - } \\
\text { band }\end{array}$} & \multicolumn{2}{|c|}{$\begin{array}{l}\text { Single training } \\
\text { image }\end{array}$} & \multicolumn{2}{|c|}{$\begin{array}{l}5 \text { training } \\
\text { images }\end{array}$} & \multicolumn{2}{|c|}{ Leave-one-out } \\
\hline & N8 & $\mathbf{N}_{4} \& \mathbf{N}_{D}$ & N8 & $\mathbf{N}_{4} \& N_{D}$ & N8 & $\mathbf{N}_{4} \& \mathbf{N}_{D}$ \\
\hline $\mathrm{LL}_{1}$ & 68.0 & 69.0 & 96.4 & 96.6 & 99.5 & 99.0 \\
\hline $\mathrm{HL}_{1}$ & 36.1 & 57.0 & 61.5 & 85.6 & 67.5 & 92.0 \\
\hline $\mathrm{LH}_{1}$ & 20.7 & 33.8 & 35.7 & 57.2 & 43.0 & 66.3 \\
\hline $\mathrm{HH}_{1}$ & 7.6 & 8.3 & 9.8 & 11.8 & 10.0 & 12.3 \\
\hline $\mathrm{LL}_{2}$ & 68.9 & 69.7 & 95.2 & 96.0 & 98.5 & 98.3 \\
\hline $\mathrm{HL}_{2}$ & 30.0 & 50.1 & 52.7 & 81.4 & 60.5 & 87.8 \\
\hline $\mathrm{LH}_{2}$ & 20.4 & 35.0 & 39.0 & 63.5 & 49.3 & 73.5 \\
\hline $\mathrm{HH}_{2}$ & 5.2 & 7.1 & 6.6 & 10.3 & 6.3 & 12.8 \\
\hline
\end{tabular}

Table 4: Recognition rates (\%) for ORL database; each wavelet sub-band is divided into $5 \times 5$ blocks

\begin{tabular}{ccccccc}
\hline $\begin{array}{c}\text { Sub - } \\
\text { band }\end{array}$ & \multicolumn{2}{c}{$\begin{array}{c}\text { Single training } \\
\text { image }\end{array}$} & \multicolumn{2}{c}{$\begin{array}{c}\mathbf{5} \text { training } \\
\text { images }\end{array}$} & \multicolumn{2}{c}{ Leave-one-out } \\
& $\mathbf{N}_{\mathbf{8}}$ & $\mathbf{N}_{\mathbf{4}}$ \& $\mathbf{N}_{\mathbf{D}}$ & $\mathbf{N}_{\mathbf{8}}$ & $\mathbf{N}_{\mathbf{4}} \& \mathbf{N}_{\mathbf{D}}$ & $\mathbf{N}_{\mathbf{8}}$ & $\mathbf{N}_{\mathbf{4}} \boldsymbol{\&} \mathbf{N}_{\mathbf{D}}$ \\
\hline $\mathrm{LL}_{1}$ & 67.9 & 67.9 & 95.4 & 96.3 & 99.0 & 98.8 \\
$\mathrm{HL}_{1}$ & 41.3 & 60.2 & 72.5 & 88.6 & 83.5 & 94.8 \\
$\mathrm{LH}_{1}$ & 27.8 & 42.8 & 49.4 & 75.2 & 57.0 & 84.5 \\
$\mathrm{HH}_{1}$ & 7.4 & 9.2 & 10.1 & 13.1 & 9.8 & 15.0 \\
\hline $\mathrm{LL}_{2}$ & 65.3 & 64.1 & 94.4 & 94.6 & 99.0 & 98.3 \\
$\mathrm{HL}_{2}$ & 33.8 & 55.7 & 63.7 & 88.1 & 75.5 & 93.8 \\
$\mathrm{LH}_{2}$ & 26.3 & 43.0 & 51.9 & 77.6 & 62.5 & 88.8 \\
$\mathrm{HH}_{2}$ & 6.1 & 10.9 & 10.2 & 18.5 & 12.3 & 24.0 \\
\hline
\end{tabular}

It is important to mention that the overall number of features obtained by the proposed four-neighbour LBP approach represent a $46 \%$ reduction of the number of features obtained by the original uniform eight-neighbour LBP. This has significant implications for real-time matching in large scale databases and biometric data hiding.

\subsubsection{Fusion of $L B P_{8}$ with combined $\mathrm{LBP}_{4}$ and $L P B_{D}$ in the} wavelet domain:

By looking at the results of the experiments reported in sec. 4.3.1, where a single sub-band was used to represent a face image, we notice that the combination of $\mathrm{LPB}_{4}$ and $\mathrm{LBP}_{\mathrm{D}}$ features achieve a higher recognition accuracy for non-LL sub-bands. On the other hand, $\mathrm{LBP}_{8}^{\mathrm{u} 2}$ performs better for $\mathrm{LL}$ 
sub-bands. For this reason, and based on previous studies that used multiple sub-bands, we evaluated a technique that combines the use of $\mathrm{LBP}_{8}^{\mathrm{u} 2}$ on LL sub-bands and $\mathrm{LPB}_{4}$ and $\mathrm{LBP}_{\mathrm{D}}$ on non-LL sub-bands. Tables 5 to 8 show recognition rates when different combination with different number of sub-bands are used as a face descriptor.

Table 5: Recognition rates $(\%)$ for Yale database; each wavelet sub-band is divided into $3 \times 3$ blocks

\begin{tabular}{|c|c|c|c|c|c|c|}
\hline \multirow{2}{*}{ Sub -bands } & \multicolumn{2}{|c|}{$\begin{array}{l}\text { Single } \\
\text { training }\end{array}$} & \multicolumn{2}{|c|}{$\begin{array}{l}5 \text { training } \\
\text { images }\end{array}$} & \multicolumn{2}{|c|}{$\begin{array}{c}\text { Leave-one- } \\
\text { out }\end{array}$} \\
\hline & $\mathbf{N}_{8}$ & $\begin{array}{c}\mathrm{N}_{4} \& \\
\mathrm{~N}_{\mathrm{D}}\end{array}$ & $\mathbf{N}_{8}$ & $\begin{array}{c}\mathrm{N}_{4} \& \\
\mathrm{~N}_{D}\end{array}$ & $\mathbf{N}_{8}$ & $\begin{array}{c}\mathbf{N}_{4} \& \\
\mathbf{N}_{D}\end{array}$ \\
\hline $\mathrm{LL}_{1}, \mathrm{HL}_{1}$ & 77.7 & 82.0 & 93.1 & 95.9 & 93.3 & 97.6 \\
\hline $\mathrm{LL}_{1}, \mathrm{LH}_{1}$ & 78.1 & 82.4 & 91.6 & 95.9 & 91.6 & 97.0 \\
\hline $\mathrm{LL}_{1}, \mathrm{HL}_{1}, \mathrm{LH}_{1}$ & 79.2 & 85.2 & 92.7 & 97.7 & 93.9 & 98.8 \\
\hline ALL Level 1 & 78.1 & 83.9 & 92.7 & 97.6 & 93.3 & 98.8 \\
\hline $\mathrm{LL}_{2}, \mathrm{HL}_{2}$ & 71.8 & 74.5 & 88.9 & 90.7 & 89.7 & 93.9 \\
\hline $\mathrm{LL}_{2}, \mathrm{LH}_{2}$ & 73.8 & 76.7 & 87.4 & 91.6 & 90.1 & 92.1 \\
\hline $\mathrm{LL}_{2}, \mathrm{HL}_{2}, \mathrm{LH}_{2}$ & 75.9 & 82.0 & 90.9 & 94.6 & 93.9 & 96.4 \\
\hline ALL Level 2 & 75.9 & 81.5 & 90.9 & 94.9 & 93.9 & 97.0 \\
\hline $\begin{array}{l}\mathrm{LL}_{1}, \mathrm{HL}_{1}, \mathrm{LH}_{1} \text {, } \\
\mathrm{LL}_{2}, \mathrm{HL}_{2}, \mathrm{LH}_{2}\end{array}$ & 80.7 & 86.4 & 93.6 & 98.1 & 93.9 & 99.4 \\
\hline
\end{tabular}

Table 6: Recognition rates $(\%)$ for Yale database; each wavelet sub-band is divided into $5 \times 5$ blocks

\begin{tabular}{|c|c|c|c|c|c|c|}
\hline \multirow{2}{*}{ Sub -bands } & \multicolumn{2}{|c|}{$\begin{array}{c}\text { Single } \\
\text { training }\end{array}$} & \multicolumn{2}{|c|}{$\begin{array}{l}5 \text { training } \\
\text { images }\end{array}$} & \multicolumn{2}{|c|}{$\begin{array}{c}\text { Leave-one- } \\
\text { out }\end{array}$} \\
\hline & $\mathbf{N}_{8}$ & $\begin{array}{c}\mathbf{N}_{4} \& \\
\mathbf{N}_{D}\end{array}$ & $\mathbf{N}_{8}$ & $\begin{array}{c}\mathbf{N}_{4} \& \\
\mathbf{N}_{D}\end{array}$ & $\mathbf{N}_{8}$ & $\begin{array}{c}\mathbf{N}_{4} \& \\
\mathbf{N}_{\mathrm{D}}\end{array}$ \\
\hline $\mathrm{LL}_{1}, \mathrm{HL}_{1}$ & 84.4 & 85.6 & 96.1 & 96.9 & 96.4 & 98.2 \\
\hline $\mathrm{LL}_{1}, \mathrm{LH}_{1}$ & 83.6 & 87.0 & 95.1 & 97.6 & 96.4 & 98.8 \\
\hline $\mathrm{LL}_{1}, \mathrm{HL}_{1}, \mathrm{LH}_{1}$ & 84.0 & 87.2 & 95.9 & 97.9 & 97.0 & 98.2 \\
\hline ALL Level 1 & 83.8 & 86.5 & 95.8 & 97.3 & 96.4 & 98.2 \\
\hline $\mathrm{LL}_{2}, \mathrm{HL}_{2}$ & 80.3 & 81.5 & 95.0 & 94.0 & 96.4 & 95.8 \\
\hline $\mathrm{LL}_{2}, \mathrm{LH}_{2}$ & 82.7 & 85.8 & 93.7 & 97.3 & 96.4 & 97.6 \\
\hline $\mathrm{LL}_{2}, \mathrm{HL}_{2}, \mathrm{LH}_{2}$ & 83.1 & 85.8 & 96.0 & 95.7 & 98.2 & 98.8 \\
\hline ALL Level 2 & 83.1 & 87.0 & 96.0 & 96.7 & 98.2 & 99.4 \\
\hline $\begin{array}{l}\mathrm{LL}_{1}, \mathrm{HL}_{1}, \mathrm{LH}_{1}, \\
\mathrm{LL}_{2}, \mathrm{HL}_{2}, \mathrm{LH}_{2}\end{array}$ & 85.0 & 88.4 & 96.2 & 98.2 & 98.2 & 98.2 \\
\hline
\end{tabular}

Table 7: Recognition rates (\%) for ORL database; each wavelet sub-band is divided into $3 \times 3$ blocks

\begin{tabular}{ccccccc}
\hline & \multicolumn{2}{c}{$\begin{array}{c}\text { Single } \\
\text { training }\end{array}$} & \multicolumn{2}{c}{5 training } & \multicolumn{2}{c}{ Leave-one- } \\
images & \multicolumn{2}{c}{ out } \\
& $\mathbf{N}_{\mathbf{8}}$ & $\begin{array}{c}\mathbf{N}_{\mathbf{4}} \boldsymbol{\&} \\
\mathbf{N}_{\mathbf{D}}\end{array}$ & $\mathbf{N}_{\mathbf{8}}$ & $\begin{array}{c}\mathbf{N}_{\mathbf{4}} \boldsymbol{\&} \\
\mathbf{N}_{\mathbf{D}}\end{array}$ & $\mathbf{N}_{\mathbf{8}}$ & $\begin{array}{c}\mathbf{N}_{\mathbf{4}} \boldsymbol{\&} \\
\mathbf{N}_{\mathbf{D}}\end{array}$ \\
\hline $\mathrm{LL}_{1}, \mathrm{HL}_{1}$ & 69.8 & 73.2 & 95.8 & 96.6 & 99.8 & 99.5 \\
$\mathrm{LL}_{1}, \mathrm{LH}_{1}$ & 68.8 & 69.5 & 96.1 & 96.1 & 99.5 & 99.5
\end{tabular}

\begin{tabular}{|c|c|c|c|c|c|c|}
\hline \multirow{2}{*}{ Sub -bands } & \multicolumn{2}{|c|}{$\begin{array}{l}\text { Single } \\
\text { training }\end{array}$} & \multicolumn{2}{|c|}{$\begin{array}{l}5 \text { training } \\
\text { images }\end{array}$} & \multicolumn{2}{|c|}{$\begin{array}{c}\text { Leave-one- } \\
\text { out }\end{array}$} \\
\hline & $\mathbf{N}_{8}$ & $\begin{array}{c}\mathbf{N}_{4} \& \\
\mathbf{N}_{D}\end{array}$ & $\mathbf{N}_{8}$ & $\begin{array}{c}\mathbf{N}_{4} \& \\
\mathbf{N}_{D}\end{array}$ & $\mathbf{N}_{8}$ & $\begin{array}{c}\mathbf{N}_{4} \& \\
\mathbf{N}_{D}\end{array}$ \\
\hline $\mathrm{LL}_{1}, \mathrm{HL}_{1}, \mathrm{LH}_{1}$ & 70.4 & 73.3 & 96.3 & 96.7 & 99.8 & 99.8 \\
\hline ALL Level 1 & 70.3 & 73.3 & 96.1 & 96.4 & 99.8 & 99.5 \\
\hline $\mathrm{LL}_{2}, \mathrm{HL}_{2}$ & 67.8 & 70.2 & 95.2 & 96.0 & 99.0 & 98.3 \\
\hline $\mathrm{LL}_{2}, \mathrm{LH}_{2}$ & 67.3 & 66.6 & 94.7 & 95.6 & 98.8 & 99.0 \\
\hline $\mathrm{LL}_{2}, \mathrm{HL}_{2}, \mathrm{LH}_{2}$ & 67.8 & 71.1 & 94.6 & 96.7 & 98.3 & 99.3 \\
\hline ALL Level 2 & 67.1 & 70.1 & 95.0 & 96.1 & 98.0 & 99.3 \\
\hline $\begin{array}{l}\mathrm{LL}_{1}, \mathrm{HL}_{1}, \mathrm{LH}_{1}, \\
\mathrm{LL}_{2}, \mathrm{HL}_{2}, \mathrm{LH}_{2}\end{array}$ & 71.6 & 75.0 & 96.4 & 98.0 & 99.8 & 99.8 \\
\hline
\end{tabular}

Table 8: Recognition rates (\%) for ORL database; each wavelet sub-band is divided into $5 \times 5$ blocks

\begin{tabular}{|c|c|c|c|c|c|c|}
\hline \multirow{2}{*}{ Sub -bands } & \multicolumn{2}{|c|}{$\begin{array}{l}\text { Single } \\
\text { training }\end{array}$} & \multicolumn{2}{|c|}{$\begin{array}{l}5 \text { training } \\
\text { images }\end{array}$} & \multicolumn{2}{|c|}{$\begin{array}{c}\text { Leave-one- } \\
\text { out }\end{array}$} \\
\hline & $\mathbf{N}_{8}$ & $\begin{array}{c}\mathbf{N}_{4} \& \\
\mathbf{N}_{D}\end{array}$ & $\mathbf{N}_{8}$ & $\begin{array}{c}\mathbf{N}_{4} \& \\
\mathbf{N}_{D}\end{array}$ & $\mathbf{N}_{8}$ & $\begin{array}{c}\mathbf{N}_{4} \& \\
\mathbf{N}_{D}\end{array}$ \\
\hline $\mathrm{LL}_{1}, \mathrm{HL}_{1}$ & 68.4 & 70.9 & 96.2 & 96.9 & 98.3 & 99.0 \\
\hline $\mathrm{LL}_{1}, \mathrm{LH}_{1}$ & 68.5 & 68.7 & 95.5 & 95.6 & 98.8 & 99.0 \\
\hline $\mathrm{LL}_{1}, \mathrm{HL}_{1}, \mathrm{LH}_{1}$ & 69.1 & 71.1 & 96.2 & 96.6 & 98.5 & 98.8 \\
\hline ALL Level 1 & 69.2 & 71.1 & 95.7 & 96.7 & 98.8 & 98.8 \\
\hline $\mathrm{LL}_{2}, \mathrm{HL}_{2}$ & 64.1 & 67.1 & 94.5 & 94.6 & 98.8 & 98.3 \\
\hline $\mathrm{LL}_{2}, \mathrm{LH}_{2}$ & 65.0 & 63.0 & 94.9 & 94.3 & 98.8 & 98.8 \\
\hline $\mathrm{LL}_{2}, \mathrm{HL}_{2}, \mathrm{LH}_{2}$ & 64.3 & 66.1 & 93.6 & 95.6 & 98.8 & 98.5 \\
\hline ALL Level 2 & 63.6 & 65.8 & 94.1 & 94.6 & 98.0 & 98.3 \\
\hline $\begin{array}{l}\mathrm{LL}_{1}, \mathrm{HL}_{1}, \mathrm{LH}_{1}, \\
\mathrm{LL}_{2}, \mathrm{HL}_{2}, \mathrm{LH}_{2}\end{array}$ & 69.5 & 71.7 & 96.3 & 96.0 & 98.8 & 98.8 \\
\hline
\end{tabular}

From tables 5 to 8 we notice that the recognition rate of the proposed method is higher in all cases when compared with results obtained $\mathrm{LBP}_{8}^{\mathrm{u} 2}$. Also, the number of features used in this experiment has fallen by $23 \%-35 \%$ of the total number when compared with that of using normal uniform 8 neighbours method. Figure 3 illustrates the size of histogram features for different LBP features. Here, the $\mathrm{X}$-axis is the number of sub-bands used to extract features and the y-axis is the number of bins required for the LBP histogram. 


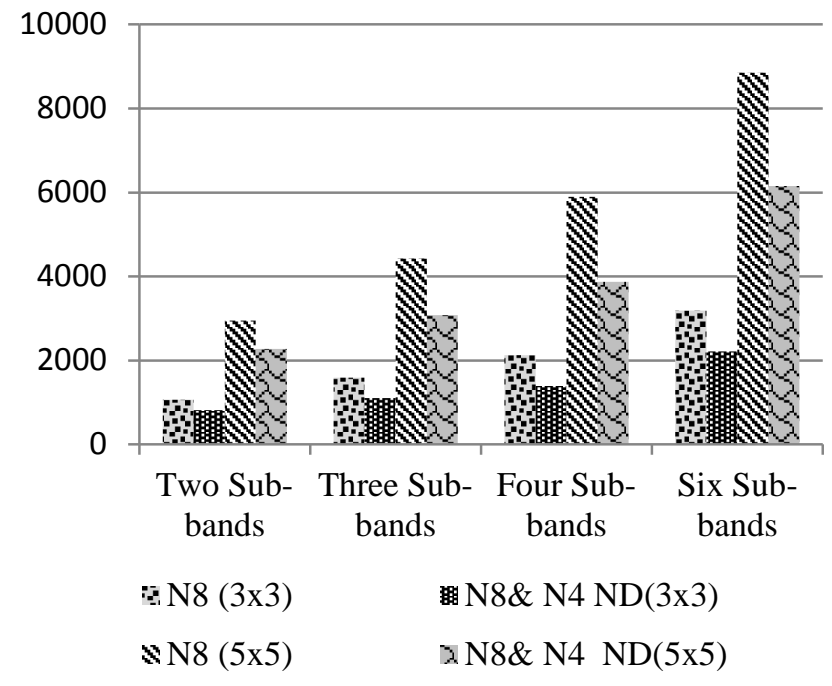

Figure 3. Number of bins used to represent face features in each case using different sub-blocking with different number of sub-bands

\section{CONCLUSIONS}

In this paper, to fully extract useful features from an image, a mutli-scale LBP is proposed. The main advantage of the proposed method is the reduced number of features that represent each block in sub-bands. This is very important for some applications like secure communication of face biometrics using steganography.

From the face recognition results we can conclude that extracting features using $\mathrm{N}_{8}$ has better recognition rate when LL sub-bands are used whilst separating 8 neighbour into two parts and extracting the LBP code from each part separately and concatenating together is suitable for non-LL sub-bands. We have demonstrated that the use of $\mathrm{N}_{8}$ for LL and $N_{4} \& N_{D}$ for non-LL sub-bands for multiple sub-bands yields higher recognition rate when compared with using $\mathrm{N}_{8}$ for all sub-bands.

\section{REFERENCES}

[1] T. Ojala, M. Pietikäinen and D. Harwood, "A Comparative Study of Texture Measures with Classification based on Featured Distributions," Pattern Recognition, vol. 29, no. 1, pp. 51-59, January 1996.

[2] Z. Guo, L. Zhang, D. Zhang and X. Mou, "Hierarchical Multiscale LBP for Face and Palmprint Recognition," in Proceedings of IEEE 17th International Conference on Image Processing, pp. 4521 - 4524, Hong Kong, September 2010.

[3] T. Ahonen, A. Hadid and M. Pietikainen, "Face Recognition with Local Binary Patterns," in Proceedings of the 9th european conference on computer vision, pp. 469 - 481, Berlin Heidelberg, 2004.
[4] T. Ojala, M. Pietikäinen and T. Mäenpää, "Multiresolution Gray Scale and Rotation Invariant Texture Classification with Local Binary Patterns," IEEE Transactions on Pattern Analysis and Machine Intelligence, vol. 24 , no. 7, pp. 971987, July 2002.

[5] Y. Wang, Q. Yan and K. Li, "Hand Vein Recognition based on Multi-scale LBP and Wavelet," in Proceedings of the 2011 International Conference on Wavelet Analysis and Pattern Recognition, pp. 214 - 218, Guilin, July 2011.

[6] W. Wang, W. Chen and D. Xu, "Pyramid-based Multi-scale LBP Features for Face Recognition," in International Conference on Multimedia and Signal Processing, pp. 151 155, Guilin, Guangxi, May 2011.

[7] X. Liu, M. Du and L. Jin, "Face Features Extraction Based on Multi-scale LBP," in 2nd International Conference on Signal Processing Systems (ICSPS), pp. V2-438 - V2-441, July 2010.

[8] H. Sellahewa and S. Jassim, "Image Quality-based Adaptive Face Recognition," IEEE Transactions on Instrumentation and Measurement, vol. 59, no. 4, pp. 805-813, April 2010.

[9] H. Tang, Y. Sun, B. Yin and Y. Ge, "Face Recognition based on Haar LBP Histogram," in 3rd International Conference on Advanced Computer Theory and Engineering (ICACTE), pp. V6-235 - V6-238, Chengdu, August 2010.

[10] T. Ahonen, A. Hadid and M. Pietikainen, "Face Description with Local Binary Patterns: Application to Face Recognition," IEEE Transactions on Pattern Analysis and Machine Intelligence, vol. 28, no. 12, pp. 2037-2041, 2006.

[11] C. Shan, S. Gong and P. McOwan, "Facial Expression Recognition based on Local Binary Patterns: A Comprehensive Study," Image and Vision Computing, vol. 27, pp. 803-816, 2009.

[12] F. S. Samaria and A. C. Harter, "Parameterisation of a Stochastic Model for Human Face Identification," in Proceedings of the 2nd IEEE Workshop on Applications of Computer Vision, pp. 138 - 142, Sarasota (Florida), December 1994.

[13] "http://cvc.yale.edu/projects/yalefaces/yalefaces.html". 